\title{
Acknowledgment of Ad Hoc Reviewers, 2020
}

The Editor and Associate Editors gratefully acknowledge the following ad hoc reviewers, who reviewed and co-reviewed* manuscripts for the Psychology of Men and Masculinities during the period between August 2019 through August 2020.

Irena Acic

Kari Adamsons

Michael Addis

Lieselotte Ahnert

William Allen

RaeAnn E. Anderson

Sheila Anderson

Tod Augusta-Scott

Ann M. Aviles

Jide Bamishigbin

Elizabeth A. Bates

Samuel T. Beasley

Elyssa C. Berney*

Erika Bocknek

Ashlee Borgkvist

Nicholas Borgogna

Lisa Bowleg

Guy Boysen

Kyle Brasil*

Rachel E. Brenner

Carolyn Brooks

Wangnan Cao

Roderick L. Carey

H. Haynes Carlson

Nicola Carone

Jennifer Carrano

Dan Cassino

Clewiston D. Challenger

Bin Bin Chen

Joan C. Chrisler

Christopher F. Clemens

Brian P. Cole

Corey L. Cook

Shauna M. Cooper

Elizabeth Cordero

Ieuan Cranswick

Joseph M. Currin

Laura Cutler

Leila Dal Santo*

Konstantinos Christos Daoultzis

Zoe Darwin

Carolyn Dayton

Pedro Henrique Berbert de Carvalho

Amanda Diekman
Murray Drummond

W. Justin Dyer

Christian Edwards

Keith Edwards

Karla Elliott

Melissa M. Ertl ${ }^{*}$

Adrienne Evans

Adam Everson*

Jay Fagan

Joshua M. Feinberg

Yanan Feng

Julianne Flanagan

William Michael Fleming

Miguel Gallardo

Matthew Genuchi

David Giles

Debbie Ging

Janelle R. Goodwill

Mellissa Gordon

Derrick M. Gordon

Anthony Greene

Scott Grether

Frederick G. Grieve

Sarah Grogan

Esmee Hanna

Tova Hartman

Alex Hassett

Patrick J. Heath

Martin Heesacker

Mary S. Himmelstein

Erin Kramer Holmes

James Hull

Sarah Hunter

Glen S. Jankowski

Yahong Jin

Adam Jowett

Joshua Katz

Gail Kaufman

Keith Allen Kline

Nikola Komlenac

Karolina Konopka

Natasza KosakowskaBerezecka
Karen Kramer

Nina Krüger

Oscar Labra

Ariadna Beata Łada

Suzanne H. Lease

Inna Levy

Linda Lin

Agnieszka Ewa Łyś

Alexandra Lysova

Andreia Machado

Jarred Martin

Tyler Mason

Catherine Massey

December Maxwell

Mark McCormack

Ryan A. McKelley

Katrina McLaughlin

Alex Melkonian*

Jane Meyrick

Brandon Miller

Roger Mills-Koonce

Lindsey L. Monteith

Tracy Morison

Gordon C. Nagayama Hall

Christopher D. Nettles

Socrates Alvares Nolasco

Julia O'Connor

Wonjung $\mathrm{Oh}$

Lior Oren

Noam Ostrander

Glen Frederick Palm

Daniel Paquette

Joshua Glenn Parmenter

Kenneth Parnell

Maria Manuela Peixoto

Jarrod Pendlebury

Jessica Perrotte

Armon Perry

Richard Petts

Danielle Piggott*

Charles Joseph Polihronakis

Elia Psouni

Travis Ray
Alexander Rice

Kate Richmond

Damien Ridge

Damien W. Riggs

Andrew J. Rizzo

Anton Roberts

Poul Rohleder

Jon Ross

Kevin Roy

Brett Scholz

Sarah Schoppe-Sullivan

Zac Eugene Seidler

Rogelio Serrano

Sarah Seymour-Smith

Kevin Shafer

Rachel Shaw

Geva Shenkman

Duncan M. Shields

Andrew P. Smiler

Brett Smith

Laura E. Sockol

Jennifer St. George

Amelia Stanley*

Howard Steele

Andrew Lee Stewart

Ottilie Stolte

Heidi Stolz

Anna Tarrant

Alexander Tatum

Gareth Terry

Andrew Thompson

Edward H. Thompson

Rich Tolman

Samantha Tornello

Cheryl Travis

Gareth Treharne

Matthew Tull

Arlene Walker

David J. Wimer

Charles Woods*

Zali Yager

Elif Dede Yildirim 